\title{
Near commuting multi-matrix models
}

\author{
Denjoe O'Connor ${ }^{a}$ and Veselin G. Filev ${ }^{b}$ \\ ${ }^{a, b}$ School of Theoretical Physics, Dublin Institute for Advanced Studies \\ 10 Burlington Road, Dublin 4, Ireland. \\ E-mail: denjoe@stp.dias.ie, vfilev@stp.dias.ie
}

\begin{abstract}
We investigate the radial extent of the eigenvalue distribution for Yang-Mills type matrix models. We show that, a three matrix Gaussian model with complex Myers coupling, to leading order in strong coupling is described by commuting matrices whose joint eigenvalue distribution is uniform and confined to a ball of radius $R=\left(\frac{3 \pi}{2 g}\right)^{1 / 3}$. We then study, perturbatively, a 3-component model with a pure commutator action and find a radial extent broadly consistent with numerical simulations.
\end{abstract}

KEYwords: Matrix Models, 1/N Expansion 


\section{Contents}

1 Introduction 1

2 Strongly coupled mass regulated two matrix model 3

2.1 Hemisphere distribution 4

2.2 Three matrix model realization and uniform distribution 6

3 The $p \geq 3$ matrix model $\quad 9$

$\begin{array}{ll}3.1 \text { Gauge fixing } & 10\end{array}$

$\begin{array}{ll}\text { 3.2 The effective action to two loops and stabilization } & 12\end{array}$

$\begin{array}{lll}3.3 & \text { Gauge dependence } & 14\end{array}$

$\begin{array}{llr}4 & \text { Discussion } & 16\end{array}$

$\begin{array}{llr}5 & \text { Acknowledgments } & 17\end{array}$

$\begin{array}{ll}\text { A Corrections at large coupling and lifting the distribution } & \mathbf{1 7}\end{array}$

$\begin{array}{lll}\text { A.1 Iterative Solution } & 18\end{array}$

A.2 "Lifting" the 1d distribution 20

$\begin{array}{ll}\text { B The Jacobian } & 21\end{array}$

C The constants \#(p) and $\tilde{\#}(p) \quad 22$

\section{Introduction}

Multi-matrix models arise in a wide variety of settings from matrix string theory [1], the IKKT model [2] (and its lower dimensional variants [3]), the BFFS and BMN models [4, 6] to the low energy dynamics of $D$-branes [7] and simple models of emergent geometry [8] and emergent gravity $[9,10]$. More recently the BMN and BFFS models have received attention in the context of fast scrambling in [11].

In the context of numerical simulations it has been observed that the 3-component Yang Mills matrix model (with pure commutator action) [12] has an eigenvalue distribution that is parabolic with radial extent $R=2.0$. It is tempting to suspect that this result should be derivable from first principals and this paper is an attempt in this direction. In part the motivation for the current paper was to attempt a theoretical estimate of the extent of the eigenvalue distribution in this simple model. Though we did not succeed in getting an exact result we succeeded in getting approximate results which are in broad agreement with the simulations.

The principal results of this paper are: 
- A derivation of the uniform ball distribution in a previously studied 3-matrix model, some of whose observables have been solved exactly.

- A demonstration that one can obtain the exact extent of the eigenvalue distribution in this 3-matrix model from an effective potential for this radial extent.

- The derivation of an effective potential to 2-loops for the pure Yang-Mills matrix model.

- We derive the measure for a rotationally invariant gauge fixing which is natural if the matrices are approximately commuting.

- Estimates for the radial extent in the 3-matrix Yang-Mills model, our estimate is that the extent of the eigenvalue distribution is $R=1.6 \pm 0.5$.

The structure of the paper is as follows:

Section 2 is dedicated to the mass regulated two matrix model. This model was introduced in [5] and arose again in the description of the low energy dynamics of $N$ $D$-branes which are close together in 4-dimensional space-time [7]. The strong coupling properties of the model were investigated in ref. [7] and in [13] it was shown that at strong coupling the model is in a commuting phase with the joint eigenvalue distribution described by a hemisphere.

In the first part of Section 2 we review the results of ref. [13] using a sightly different approach. In particular we split the matrices into their diagonal and off-diagonal elements and consider a general axial gauge which is equivalent to diagonalizing one of the matrices. We integrate out the perpendicular modes and obtain an effective action for the diagonal components of the matrices. Next we consider a coarse grained approximation and show that the longitudinal part of the diagonal modes has a parabolic eigenvalue distribution [13].

In the second part of Section 2 we consider an $S O(3)$ invariant non-Hermitian three matrix model constructed from a Hermitian mass term and an anti-Hermitian Myers term [14]. Integrating out one of the matrices one recovers the $S O(2)$ invariant two matrix model considered in the first part of Section 2. However, we study the model directly in the three matrix model realization by splitting the matrices into their diagonal and offdiagonal components and consider a general axial gauge for the off-diagonal modes. Next we integrate out the perpendicular modes and obtain a one-loop effective action (exact in the axial gauge) for the longitudinal part of the diagonal components. We average over all possible orientations of the constant unit vector specifying the axial gauge and obtain a three dimensional $S O(3)$ invariant effective action for the diagonal modes. Next we study the strongly coupled regime of the model and show that to leading order the ground state is described by a uniform joint eigenvalue distribution inside a solid ball. The hemisphere and parabolic distributions conjectured in ref. [13] can then be obtained by integrating the uniform distribution over one or two of the three coordinates respectively. Further, by conjecturing a uniform joint eigenvalue distribution, we obtain an effective potential for the radius of the distribution, which in the strong coupling limit recovers the exact radial extent of the distribution. It is this uniform joint eigenvalue distribution of the 
model which motivates the studies presented in Section 3 of the paper. We establish in Appendix A.2 that there is a unique lift of a one dimensional distribution to a rotationally invariant three dimensional distribution, and therefore the unique lift of the parabola is the uniform distribution. This can further be reduced to a unique rotationally invariant two dimensional distribution. Also, we develop the first order corrections to the leading large $g$ behaviour in Appendix A.2 and verify that they are sufficient to capture the next term in a large $g$ expansion of an observable whose exact result is know.

Section 3 of our work explores the properties of the $S O(p)$ invariant Hermitian pmatrix model corresponding to a commutator squared term, i.e. a pure Yang-Mills type matrix model. This family of models have been studied both numerically and in a large $p$ expansion in [15]. Special attention was given to the $p=3$ case in ref. [16-20], including the effect of finite mass and Myers terms. In particular it has been shown that, as the strength of the Myers coupling is varied, the ground state of the model undergoes an exotic phase transition from a fuzzy two sphere to a matrix phase [8]. It is this matrix phase that we explore in Section 3.

Section 3 contains four subsections. In first part of the Section 3 we outline a change of coordinates involving splitting the matrices to diagonal and off-diagonal components. The change of coordinates that we consider is also equivalent to an $S O(p)$ invariant gauge fixing condition for the off-diagonal elements. We calculate the corresponding Jacobian to second order in the off-diagonal modes. This takes into account contribution of the corresponding ghost modes to two loops. At tree level the ghost determinant is a generalized $S O(p)$ invariant Vandermonde type determinant.

The second part of Section 3 derives the effective action for the diagonal modes. By setting up a systematic perturbative expansion we obtain a two loop effective action for the diagonal elements of the model and under the assumption of an $S O(p)$ invariant distribution for these modes, within a ball of radius $R$, we obtain an effective potential for the radial extent of their distribution. We show that the radial extent of these modes stabilizes in this approximation. For the three matrix model assuming a uniform distribution inside a solid ball, which by appendix A.2 is the unique rotational invariant lift of a parabolic one dimensional distribution, we obtain an analytic estimate of the radius of the distribution.

The third part of Section 3 studies the gauge dependence of our perturbative expansion. We consider a standard Faddeev-Popov gauge fixing procedure and compare the results obtained in Feynman and Landau gauges. We argue that for a generic gauge the joint distribution of the diagonal modes will differ from the genuine joint distribution and that the Landau gauge is the most suitable for studying the model.

Section 4 of our paper is our concluding section. We summarize our results and discuss possible directions for future studies.

\section{Strongly coupled mass regulated two matrix model}

Let us consider the Hermitian two matrix model first considered by Hoppe [5]:

$$
\mathcal{Z}=\int \mathcal{D} X \mathcal{D} Y e^{-N \operatorname{tr}\left(X^{2}+Y^{2}-g^{2}[X, Y]^{2}\right)} .
$$


The matrix model (2.1) arose again in the work of Kazakov et al [7] where the partition function for large $g$ and a fresh derivation of Hoppe's solution for the observable $<\frac{\operatorname{tr}}{N}\left(X^{2}\right)>$ are given. The solution for these quantities are given in a rather complex form, which makes a more complete analysis of the theory (valid for large $g \gg 1$ ) difficult. A more detailed understanding of the model was initiated in ref. [13] where it was suggested that the joint eigenvalue distribution of the commuting saddle is given by a hemisphere distribution. The analysis of ref. [13] shows that at large coupling the eigenvalues of a single matrix are described by a parabolic eigenvalue distribution. The authors then argue that this suggests a hemisphere eigenvalue distribution for the commuting saddle of the two matrix model.

\subsection{Hemisphere distribution}

Let us review the results of ref. [13], by following a slightly different approach which we find instructive. In particular we split the matrices to diagonal and off-diagonal components and integrate out the off-diagonal modes by imposing an axial gauge condition. To begin with let us define:

$$
X_{i j}=x_{i}^{1} \delta_{i j}+a_{i j}^{1} ; \quad Y_{i j}=x_{i}^{2} \delta_{i j}+a_{i j}^{2} ; \quad \vec{x}_{i}=\left(x_{i}^{1}, x_{i}^{2}\right) ; \quad \vec{a}_{i j}=\left(a_{i j}^{1}, a_{i j}^{2}\right) .
$$

The action in (2.1) can be written as:

$$
S[X, Y]=\sum_{i} \vec{x}_{i}^{2}+\sum_{i \neq j}\left|\vec{a}_{i j}\right|^{2}+g^{2} \sum_{i \neq j}\left|\left(x_{i}^{\mu}-x_{j}^{\mu}\right) a_{i j}^{\nu} \epsilon_{\mu \nu}+\left(a^{\mu} a^{\nu}\right)_{i j} \epsilon_{\mu \nu}\right|^{2} ; \quad \mu, \nu=1,2 .
$$

Let us also consider a constant unit vector $\vec{n}=\left(n^{1}, n^{2}\right)$ and define:

$$
\vec{a}^{\|}=\vec{n}(\vec{n} \cdot \vec{a}) ; \quad \vec{a}^{\perp}=(\hat{1}-\vec{n} \vec{n}) \cdot \vec{a} .
$$

Now we can use the $S U(N)$ symmetry of the matrix model to fix the gauge:

$$
\vec{n} \cdot \vec{a}_{i j}=0
$$

Note that in this gauge the action is quadratic in the off-diagonal modes and hence the first loop effective action is exact! Indeed, without loss of generality we can consider $n^{1} \neq 0$ and express:

$$
a^{1}=-\frac{n^{2}}{n^{1}} a^{2}
$$

It is then easy to check that:

$$
a^{\mu} a^{\nu} \epsilon_{\mu \nu}=\left[a^{1}, a^{2}\right]=-\frac{n^{2}}{n^{1}}\left[a^{2}, a^{2}\right]=0 .
$$

This simplification is not a surprise since the gauge condition (2.5) with a constant $\vec{n}$ is equivalent to diagonalizing one of the matrices. The simplified action can be written as:

$$
S\left[X^{\mu}\right]=\sum_{i}\left[\left(\vec{n} \cdot \vec{x}_{i}\right)^{2}+\vec{x}_{i}^{\perp 2}\right]+\sum_{i \neq j}\left[1+g^{2}\left(\vec{n} \cdot\left(\vec{x}_{i}-\vec{x}_{j}\right)\right)^{2}\right]\left|\vec{a}_{i j}^{\perp}\right|^{2}+S_{\mathrm{FP}}
$$


Here $S_{F P}$ is the contribution from the Faddeev-Popov determinant resulting from the gauge choice (2.5). We have also split the diagonal modes into longitudinal $\vec{n} . \vec{x}$ and perpendicular $\vec{x}_{i}^{\perp}$ components. It is easy to verify that:

$$
S_{F P}=-\frac{1}{2} \sum_{i \neq j} \log \left(\vec{n} \cdot\left(\vec{x}_{i}-\vec{x}_{j}\right)\right)^{2} .
$$

Note that if we choose a basis in which $\vec{n}=(1,0)$ or $\vec{n}=(0,1)$ the Faddeev-Popov determinant is just the standard Vandermonde determinant. It is now straightforward to integrate out the perpendicular elements of the matrices. The resulting effective action (we divide by $N^{2}$ ) for the longitudinal diagonal modes is:

$$
S_{\text {eff }}[\vec{x}]=\frac{1}{N} \sum_{i}\left(\vec{n} \cdot \vec{x}_{i}\right)^{2}-\frac{1}{2 N^{2}} \sum_{i \neq j} \log \left[\frac{\left(\vec{n} \cdot\left(\vec{x}_{i}-\vec{x}_{j}\right)\right)^{2}}{1+g^{2}\left(\vec{n} \cdot\left(\vec{x}_{i}-\vec{x}_{j}\right)\right)^{2}}\right] .
$$

A few comments are in order: Note that the first loop effective action (2.10) is exact in this gauge. It is also valid for all $g^{2}$. However, we know that the matrices commute only in the large $g^{2}$ limit, so the meaning of the individual components of $\vec{x}_{i}$ needs clarification. Our strategy will be to lift the eigenvalue distribution of the matrix $\vec{n} \cdot \vec{X}$ specified by (2.10) to a rotationally invariant 2-dimensional distribution which in the large $g$ limit becomes the joint eigenvalue distribution for the commuting eigenvalues of $X$ and $Y$. See A.2 for a more detailed discussion.

In this spirit we consider a coarse grained description and extremize the following functional:

$$
\begin{aligned}
S_{\mathrm{eff}}[\rho(\vec{x})] & =\int d^{2} x \rho(\vec{x})(\vec{n} \cdot \vec{x})^{2}-\frac{1}{2} \iint d^{2} x d^{2} x^{\prime} \rho(\vec{x}) \rho\left(\vec{x}^{\prime}\right) \log \left[\frac{\left(\vec{n} \cdot\left(\vec{x}-\vec{x}^{\prime}\right)\right)^{2}}{1+g^{2}\left(\vec{n} \cdot\left(\vec{x}-\vec{x}^{\prime}\right)\right)^{2}}\right]+ \\
& +\mu\left(\int d^{2} x \rho(\vec{x})-1\right)
\end{aligned}
$$

Upon variation with respect to $\rho$ we obtain ${ }^{1}$ the equation:

$$
\mu+(\vec{n} \cdot \vec{x})^{2}=\int d^{2} x^{\prime} \rho\left(\vec{x}^{\prime}\right) \log \left[\frac{\left(\vec{n} \cdot\left(\vec{x}-\vec{x}^{\prime}\right)\right)^{2}}{1+g^{2}\left(\vec{n} \cdot\left(\vec{x}-\vec{x}^{\prime}\right)\right)^{2}}\right] .
$$

Now we can apply the following differential operator $\vec{n} . \nabla_{\vec{x}}$ to both hand sides of equation (2.12). The result is:

$$
\vec{n} \cdot \vec{x}=\int \frac{d^{2} x^{\prime} \rho\left(\vec{x}^{\prime}\right)}{\left(\vec{n} \cdot\left(\vec{x}-\vec{x}^{\prime}\right)\right)\left(1+g^{2}\left(\vec{n} \cdot\left(\vec{x}-\vec{x}^{\prime}\right)\right)^{2}\right)}
$$

This is the equation that we can use to deduce the form of the joint eigenvalue distribution $\rho(\vec{x})$. Note that this is almost equation (5) from ref. [13]. To make the analogy complete

\footnotetext{
${ }^{1}$ If we average over $n$ here we obtain

$$
\mu+\frac{\vec{x}^{2}}{2}=2 \int d^{2} x^{\prime} \rho\left(\overrightarrow{x^{\prime}}\right) \ln \left(\frac{\mid \vec{x}-\overrightarrow{x^{\prime} \mid}}{1+\sqrt{1+g^{2}\left|\vec{x}-\vec{x}^{\prime}\right|^{2}}}\right) .
$$
}


we define $u=\vec{n} \cdot \vec{x}$ and choose a coordinate frame in the integral along $\vec{x}^{\prime}$ in which we have $\vec{n} \cdot \vec{x}^{\prime}=x^{\prime 1}$.

$$
u=\int \frac{d x^{\prime 1} \rho_{1}\left(x^{\prime 1}\right)}{\left(u-x^{\prime 1}\right)\left(1+g^{2}\left(u-x^{\prime 1}\right)^{2}\right)}
$$

Note that we have defined:

$$
\rho_{1}\left(x^{1}\right)=\int d x^{2} \rho\left(x^{1}, x^{2}\right)
$$

We next observe that for large $g$ :

$$
\frac{1}{2} \log \left[\frac{g^{2} x^{2}}{1+g^{2} x^{2}}\right]=-\frac{\pi}{g} \delta(x)+O\left(1 / g^{2}\right) \quad \text { and } \quad \frac{1}{x\left(1+g^{2} x^{2}\right)}=-\frac{\pi}{g} \delta^{\prime}(x)+O\left(1 / g^{2}\right),
$$

Substituting in equation (2.14) we obtain:

$$
u=-\frac{\pi}{g} \int d x^{\prime 1} \rho_{1}\left(x^{1}\right) \delta^{\prime}\left(u-x^{1}\right)+O\left(1 / g^{2}\right)=-\frac{\pi}{g} \rho_{1}^{\prime}(u)+O\left(1 / g^{2}\right) .
$$

Next we solve equation (2.17) to leading order in $g^{-1}$ and normalize $\int d u \rho_{1}(u)=1$. The resulting distribution is given by [13]:

$$
\rho_{1}\left(x^{1}\right)=\frac{3}{4 R^{3}}\left(R^{2}-x^{1^{2}}\right)
$$

with

$$
R=\left(\frac{3 \pi}{2 g}\right)^{1 / 3} .
$$

Now the definition of $\rho_{1}$ from equation (2.15) and the $S O(2)$ symmetry of the distribution together with Appendix A.2 gives the unique solution for $\rho_{2}\left(x^{1}, x^{2}\right)$ :

$$
\rho_{2}\left(x^{1}, x^{2}\right)=\frac{3 \sqrt{R^{2}-\vec{x}^{2}}}{2 \pi R^{3}}
$$

as originally suggested in [13]. Equation (2.20) is the desired hemisphere distribution reported in ref. [13]. Note that the derivation of the two-dimensional distribution (2.20) is somewhat indirect. Indeed what we derived from the effective action (2.10) was the one-dimensional parabolic distribution (2.18). In the next subsection we will consider a three matrix model equivalent to the two matrix model (2.1) and prove directly that at strong coupling the corresponding three dimensional eigenvalue distribution is an uniform distribution inside a solid ball. The two dimensional hemisphere eigenvalue distribution can be obtained by integrating out one of the eigenvalues.

\subsection{Three matrix model realization and uniform distribution}

Let us now consider the model (also originally introduced parenthetically by Hoppe [5] page 73 and further discussed in [7]):

$$
\mathcal{Z}=\int \mathcal{D} X \mathcal{D} Y \mathcal{D} Z e^{-N \operatorname{tr}\left(\mathrm{X}^{2}+\mathrm{Y}^{2}+\mathrm{Z}^{2}-\mathrm{i} \alpha[\mathrm{X}, \mathrm{Y}] \mathrm{Z}\right)} .
$$


It is easy to verify that if one integrates out the $Z$ matrix and defines $g^{2}=(i \alpha)^{2} / 4$ one recovers the two matrix model (2.1). This suggests that the model (2.21) should be as solvable as the two matrix model. Note also that there is a global $S O(3)$ symmetry rotating the $X, Y$ and $Z$ matrices. We find it instructive to analyze the model in the spirit described in section 2.1. To begin with let us define:

$$
X_{i j}=x_{i}^{1} \delta_{i j}+a_{i j}^{1} ; \quad Y_{i j}=x_{i}^{2} \delta_{i j}+a_{i j}^{2} ; \quad Z_{i j}=x_{i}^{3} \delta_{i j}+a_{i j}^{3} ; \quad \vec{x}_{i}=\left(x_{i}^{1}, x_{i}^{2}, x^{3}\right) ; \quad \vec{a}_{i j}=\left(a_{i j}^{1}, a_{i j}^{2}, a_{i j}^{3}\right) .
$$

Next we consider a constant unit vector $\vec{n}=\left(n^{1}, n^{2}, n^{3}\right)$, define:

$$
\vec{x}^{\perp}=(\hat{1}-\vec{n} \vec{n}) \cdot \vec{x} ; \quad \vec{a}^{\|}=\vec{n}(\vec{n} \cdot \vec{a}) ; \quad \vec{a}^{\perp}=(\hat{1}-\vec{n} \vec{n}) \cdot \vec{a} .
$$

and impose the axial gauge $\vec{n} \cdot \vec{a}=0$. The action in (2.21) can then be written as:

$$
S[\vec{x}, \vec{a}]=\sum_{i}\left[\left(\vec{n} \cdot \vec{x}_{i}\right)^{2}+\vec{x}_{i}^{\perp 2}\right]+\sum_{i \neq j} a_{i j}^{\mu \perp}\left[\delta^{\mu \nu}-i \frac{\alpha}{2} \epsilon_{\mu \nu \rho} n^{\rho}\left(\vec{n} \cdot \vec{\Delta}_{i j}\right)\right] a_{j i}^{\nu \perp},
$$

where we have defined $\vec{\Delta}_{i j}=\vec{x}_{i}-\vec{x}_{j}$. Note that there is no term cubic in $\vec{a}$ in the action (2.24), because of the axial gauge. This means that the one loop effective action is exact. Now we proceed as in section 2.1 and integrate the perpendicular matrix elements. One can show that the resulting effective action is given by:

$$
S_{\mathrm{eff}}[(\vec{n} \cdot \vec{x})]=\frac{1}{N} \sum_{i}\left(\vec{n} \cdot \vec{x}_{i}\right)^{2}-\frac{1}{2 N^{2}} \sum_{i \neq j}^{N} \log \left[\frac{g^{2}\left(\vec{n} \cdot\left(\vec{x}_{i}-\vec{x}_{j}\right)\right)^{2}}{1+g^{2}\left(\vec{n} \cdot\left(\vec{x}_{i}-\vec{x}_{j}\right)\right)^{2}}\right]+\frac{(N-1)}{2 N} \log g^{2},
$$

where $g^{2}=(i \alpha)^{2} / 4$. Next we consider a coarse grained approximation and vary the corresponding distribution function $\rho$ to obtain the equation:

$$
\mu+(\vec{n} \cdot \vec{x})^{2}=\int d^{3} x^{\prime} \rho\left(\vec{x}^{\prime}\right) \log \left[\frac{g^{2}\left(\vec{n} \cdot\left(\vec{x}-\vec{x}^{\prime}\right)\right)^{2}}{1+g^{2}\left(\vec{n} \cdot\left(\vec{x}-\vec{x}^{\prime}\right)\right)^{2}}\right] .
$$

Note that equation (2.26) is valid for any choice of $\vec{n}$. Next we average over all possible directions that $\vec{n}$ can take with a uniform weight. Or equivalently average over the unit two-sphere. It is easy to show that:

$$
\frac{1}{4 \pi} \int d \Omega_{2}(\vec{n} \cdot \vec{x})^{2}=\frac{1}{3} \vec{x}^{2} .
$$

The right-hand side of equation (2.26) requires a bit more careful analysis. One can show that:

$$
\begin{aligned}
& J\left(g\left|\vec{x}-\overrightarrow{x^{\prime}}\right|\right):=\frac{1}{4 \pi} \int d \Omega_{2} \log \left[\frac{g^{2}\left(\vec{n} \cdot\left(\vec{x}-\vec{x}^{\prime}\right)\right)^{2}}{1+g^{2}\left(\vec{n} \cdot\left(\vec{x}-\vec{x}^{\prime}\right)\right)^{2}}\right]=\frac{1}{2 g\left|\vec{x}-\vec{x}^{\prime}\right|} \int_{-g\left|\vec{x}-\vec{x}^{\prime}\right|}^{g\left|\vec{x}-\vec{x}^{\prime}\right|} d \eta \log \left(\frac{\eta^{2}}{1+\eta^{2}}\right) \\
& =\frac{-2 \arctan \left(g\left|\vec{x}-\vec{x}^{\prime}\right|\right)}{g\left|\vec{x}-\vec{x}^{\prime}\right|}+\log \left[\frac{g^{2}\left(\vec{x}-\vec{x}^{\prime}\right)^{2}}{1+g^{2}\left(\vec{x}-\vec{x}^{\prime}\right)^{2}}\right]=-\frac{\pi}{g} \frac{1}{\left|\vec{x}-\vec{x}^{\prime}\right|}+O\left(\frac{1}{g^{2}}\right),
\end{aligned}
$$


where the term $O\left(g^{-2}\right)$ should be thought of as the difference of the lefthand side of equation (2.28) and the $g^{-1}$ term on the righthand side of the equation. To leading order in $1 / g$ equation (2.26) becomes:

$$
\mu+\frac{1}{3} \vec{x}^{2}=-\frac{\pi}{g} \int d^{3} x^{\prime} \rho\left(\vec{x}^{\prime}\right) \frac{1}{\left|\vec{x}-\vec{x}^{\prime}\right|} .
$$

Next we apply the Laplacian $\Delta_{x}$ on both hand-sides of equation (2.29) to obtain:

$$
2=\frac{4 \pi^{2}}{g} \int d^{3} x^{\prime} \rho\left(\vec{x}^{\prime}\right) \delta\left(\vec{x}-\vec{x}^{\prime}\right)=\frac{4 \pi^{2}}{g} \rho(\vec{x}) .
$$

Therefore we conclude that:

$$
\rho(\vec{x})=\frac{g}{2 \pi^{2}}=\text { const }
$$

and hence at strong coupling we get a uniform eigenvalue distribution inside a ball. In order to estimate the radius of the eigenvalue distribution $R$ we use the normalization of $\rho(\vec{x})$ :

$$
\int d^{3} x \rho(\vec{x})=\frac{4 \pi}{3} R^{3} \rho=1 .
$$

From equations (2.31) and (2.32) we obtain:

$$
R=\left(\frac{3 \pi}{2 g}\right)^{1 / 3}
$$

which is exactly the radius obtained in ref. [13] reported in equation (2.19). It is also straightforward to obtain the hemisphere distribution (2.20). Indeed:

$$
\rho_{2}\left(x^{1}, x^{2}\right)=\int_{-\sqrt{R^{2}-x^{1^{2}}-x^{2^{2}}}}^{\sqrt{R^{2}-x^{1^{2}}-x^{2}}} d x^{3} \rho\left(x^{1}, x^{2}, x^{3}\right)=\frac{3 \sqrt{R^{2}-x^{1^{2}}-x^{2^{2}}}}{2 \pi R^{3}} .
$$

Some additional comments are worthwhile here. First one can push the analysis further by observing that if we don't assume large coupling instead of (2.29) we obtain

$$
\mu+\frac{1}{3} \vec{x}^{2}=\int d^{3} x^{\prime} \rho\left(\vec{x}^{\prime}\right) J\left(g\left|\vec{x}-\overrightarrow{x^{\prime}}\right|\right) .
$$

which upon acting with the Laplacian and noting

$$
\nabla^{2} J(x)=\frac{2}{\left(1+g^{2}\left|\vec{x}-\overrightarrow{x^{\prime}}\right|^{2}\right)\left|\vec{x}-\overrightarrow{x^{\prime}}\right|^{2}}
$$

we obtain the integral equation

$$
1=\int d^{3} x^{\prime} \frac{\rho\left(\vec{x}^{\prime}\right)}{\left(1+g^{2}\left|\vec{x}-\overrightarrow{x^{\prime}}\right|^{2}\right)\left|\vec{x}-\overrightarrow{x^{\prime}}\right|^{2}}
$$

whose large $g$ behaviour is

$$
\frac{1}{\left(1+g^{2}\left|\vec{x}-\overrightarrow{x^{\prime}}\right|^{2}\right)\left|\vec{x}-\overrightarrow{x^{\prime}}\right|^{2}}=\frac{\pi}{2 g} \frac{\delta\left(\left|\vec{x}-\overrightarrow{x^{\prime}}\right|\right)}{\left|\vec{x}-\overrightarrow{x^{\prime}}\right|^{2}}+O\left(1 / g^{2}\right)=\frac{2 \pi^{2}}{g} \delta\left(\vec{x}-\overrightarrow{x^{\prime}}\right)+O\left(1 / g^{2}\right)
$$


So to leading order we obtain $\rho(x)=\frac{g}{2 \pi^{2}} \theta(R-r)$.

Given that the eigenvalue distribution is concentrated in the interior of a ball of radius $R$ we can further deduce that this radius is determined by

$$
g^{2}=\frac{2}{3 \pi}(g R)^{3}+\cdots \quad \text { and that } \quad \nu=\frac{g^{2}}{3} \int \rho(\vec{x}) \vec{x}^{2}=\frac{(g R)^{2}}{5}+\ldots
$$

from which we find to leading order in large $g$ the observable

$$
\nu=\frac{(12 \pi)^{2 / 3}}{20} g^{4 / 3}+\cdots
$$

in agreement with [7].

So far our considerations concerned only the leading order behaviour of the distribution at large $g$. However, to verify the stability of our result we have to explore the next order corrections to the uniform distribution (2.31). It turns out that one can obtain an integral equation of the second kind for the correction to the uniform distribution $\Delta \rho$ in terms of a kernel

$$
\Delta K\left(\vec{x}-\overrightarrow{x^{\prime}}\right)=\frac{g}{4 \pi^{2}} J\left(\vec{x}-\overrightarrow{x^{\prime}}\right)-\delta\left(\vec{x}-\overrightarrow{x^{\prime}}\right) .
$$

This equation can be developed recursively in a series providing a perturbative expansion of the corrections to the uniform distribution at large g. We refer the reader to Appendix A.1 for a detailed derivation of the recursive series and calculation of the next to leading order correction to the distribution. In this appendix we also check our result by calculating the next to leading order term in the large $g$ expansion of the observable $\nu$ defined in (2.39). Again our results agree with the results of [7], see equation (A.15).

Further: Let us provide an alternative derivation of the radius of the distribution (2.33). If we average over $\vec{n}$ the effective action (2.25), assume a constant eigenvalue distribution within a sphere of radius $R$ i.e. $\rho(r)=\frac{3 \theta(R-r)}{4 \pi R^{3}}$ and use

$$
\int d^{3} x d^{3} x^{\prime} \frac{\rho(x) \rho\left(x^{\prime}\right)}{\left|\vec{x}-\vec{x}^{\prime}\right|}=\frac{6}{5 R}
$$

to leading order in $1 / g$ we obtain:

$$
S_{\text {eff }}=V_{\text {eff }}(R)=\frac{R^{2}}{5}+\frac{3 \pi}{5 g R} .
$$

Varying with respect to $R$ we find that $R^{3}=\frac{3 \pi}{2 g}$ in agreement with the exact expression (2.19). In the next section we attempt to use such an effective potential (derived perturbatively) to estimate the extent of the eigenvalue distribution in a three matrix model with pure commutator action.

\section{$3 \quad$ The $p \geq 3$ matrix model}

In this section we consider the p-matrix model:

$$
S[X]=N \operatorname{Tr}\left(-\frac{1}{4}\left[X^{\mu}, X^{\nu}\right]^{2}\right) ; \quad \mu, \nu=1 \ldots p ;
$$


where $X_{a}$ are Hermitian $N \times N$ matrices. The partition function is given by:

$$
\mathcal{Z}=\int d X_{a} e^{-S[X]}=\int \mathcal{D} X e^{N \frac{1}{4} \operatorname{Tr}\left[X^{\mu}, X^{\nu}\right]^{2}}
$$

Note that in addition to the $S U(N)$ gauge invariance the model (3.2) has a global $S O(p)$ symmetry transforming the matrices $X^{\mu}$. We are interested in the eigenvalue distribution of one of the matrices and in particular in the extent $R$ of the eigenvalue distribution in the large $N$ limit. We find it convenient to split the degrees of freedom to diagonal and off-diagonal contributions:

$$
X_{i j}^{\mu}=x_{i}^{\mu} \delta_{i j}+a_{i j}^{\mu} .
$$

In terms of the new variables the action in (3.2) can be written as:

$$
S[X]=N \frac{1}{2} \sum_{i \neq j}\left|\vec{\Delta}_{i j}\right|^{2} a_{i j}^{\mu} \Pi_{i j}^{\mu \nu} a_{j i}^{\nu}-N \sum_{i \neq j} \Delta_{i j}^{\mu} a_{i j}^{\nu}\left[a^{\mu}, a^{\nu}\right]_{j i}-N \frac{1}{4} \operatorname{tr}\left[a^{\mu}, a^{\nu}\right]^{2},
$$

where we have defined:

$$
\Delta_{i j}^{\mu}=x_{i}^{\mu}-x_{j}^{\mu} ; \quad n_{i j}^{\mu}=\Delta_{i j}^{\mu} /\left|\vec{\Delta}_{i j}\right| ; \quad \Pi_{i j}^{\mu \nu}=\delta^{\mu \nu}-n_{i j}^{\mu} n_{i j}^{\nu} ; .
$$

A standard way to proceed would be to integrate out the off-diagonal degrees of freedom $a_{i j}^{\mu}$ and obtain an effective action for the diagonal components $x_{i}^{\mu}$. Note that the quadratic term in $a^{\mu}$ in equation (3.4) is proportional to a projector and hence cannot be directly inverted. A gauge fixing is required. We find it natural to work in a gauge in which the longitudinal modes are removed, more precisely we impose the gauge fixing condition $\vec{n}_{i j} \cdot \vec{a}_{i j}=0$. In the next subsection we briefly present the change of variables necessary to implement our gauge fixing condition. We refer the reader to Appendix B for a more detailed calculation of the corresponding Jacobian.

\subsection{Gauge fixing}

Our goal is to perform a change of coordinates which is manifestly $S O(p)$ invariant and convenient in calculating quantum corrections to the effective potential governing the ground state of the theory.

Let us consider a set of $p-1$ Hermitian matrices $a_{i j}^{m \perp}(m=1 \ldots p-1 ; i, j=1 \ldots N)$ with vanishing diagonal components $\left(a_{i i}^{m \perp}=0\right)$. A slightly more general way to parametrize this set of matrices is to consider a set of $p$ linearly dependent matrices $a_{i j}^{\mu \perp}(\mu=1 \ldots p)$ satisfying:

$$
\sum_{\mu=1}^{p} n^{\mu} \cdot a^{\mu \perp} \equiv \vec{n} \cdot \vec{a}^{\perp}=0,
$$

where $\vec{n}$ is a constant $p$-dimensional unit vector and we have suppressed the indices $i, j$. Next let us consider any set of $N^{2}-N$ orthogonal matrices $R_{i j} \in S O(p)$ satisfying:

$$
\begin{aligned}
& R_{i j} \cdot \vec{n}=\vec{n}_{i j} \quad \text { for } \quad i>j ; \\
& R_{i j}=R_{j i} \quad \text { for } \quad i<j ;
\end{aligned}
$$


Clearly such a set of matrices always exists. Now we define the Hermitian matrices:

$$
\vec{a}_{i j} \equiv R_{i j} \cdot \vec{a}_{i j}^{\perp} \quad \text { for } \quad i \neq j \quad \text { and } \quad \vec{a}_{i i} \equiv 0 ; .
$$

The matrices $\vec{a}_{i j}$ are linearly dependent and satisfy the properties:

$$
\begin{aligned}
& \vec{n}_{i j} \cdot \vec{a}_{i j}=0 \\
& \vec{a}_{i j} \cdot \vec{a}_{j i}=\vec{a}_{i j}^{\perp} \cdot \vec{a}_{j i}^{\perp}
\end{aligned}
$$

Next we define the change of coordinates:

$$
X^{\mu}=U\left(x^{\mu}+a^{\mu}\right) U^{-1} ; \quad U \in S U(N) ; .
$$

Note that on the left hand-side of equation (3.11) we have a set of $p N \times N$ Hermitian matrices spanning a $p N^{2}$ dimensional linear space. On the other side by construction there are only $p-1$ linearly independent matrices labeled by $a^{\mu}$ and hence the dimension of the linear space spanned by $x^{\mu}+a^{\mu}$ is equal to $(p-1)\left(N^{2}-N\right)+p N$. This suggests that in order to have a well defined change of coordinates in equation (3.11) we need $N^{2}-N$ degrees of freedom, parameterizing the orbit of $x^{\mu}+a^{\mu}$ under the $S U(N)$ group. However a general element of $S U(N)$ has $N^{2}-1$ degrees of freedom. The $N-1$ degrees of freedom that are left out correspond to the stabilizer of the action of $S U(N)$ and as one can check are generated by a Cartan subalgebra of $s u(N)$.

It is a straightforward exercise to compute the corresponding Jacobian. The expression that one obtains is given by (we refer the reader to Appendix B for a detailed computation):

$$
J=\left(\prod_{i \neq j}\left|\vec{\Delta}_{i j}\right|\right) \operatorname{det}\left\|\delta_{i}^{l} \delta_{j}^{m}+Y_{i j}^{l m}\right\| \operatorname{det}|| \frac{\delta \theta_{r s}}{\delta u_{l m}} \|
$$

where $Y_{i j}^{l m}$ is given by:

$$
Y_{i j}^{l m}=\frac{\vec{n}_{i j}}{\left|\vec{\Delta}_{i j}\right|} \cdot\left(\vec{a}_{i l} \delta_{j}^{m}-\vec{a}_{m j} \delta_{i}^{l}\right)+\frac{\vec{a}_{i j} \cdot \Pi_{i j} \cdot \vec{a}_{m l}}{\left|\vec{\Delta}_{i j}\right|^{2}} \cdot\left(\delta_{i}^{m}-\delta_{i}^{l}-\delta_{j}^{m}+\delta_{j}^{l}\right) .
$$

Note that the last determinant in equation (3.12) is the Haar measure of $S U(N)$. Now we can write down the measure in the path integral (3.2) in terms of the new variables. To second order in the off-diagonal elements $\vec{a}_{i j}$ we have the expression:

$$
\mu=D U \prod_{i} d x_{i}\left(\prod_{i \neq j}\left|\vec{\Delta}_{i j}\right|\right) \prod_{i>j}\left(d^{p-1} a_{i j}^{\perp} d^{p-1} a_{j i}^{\perp}\right)\left\{1-2 \sum_{i \neq j} \vec{a}_{i j} \cdot \hat{D}_{i j} \cdot \vec{a}_{j i}+O\left(a^{3}\right)\right\},
$$

where $D U$ is the Haar measure of $S U(N)$ and $\hat{D}_{i j}$ is given by:

$$
D_{i j}^{\mu \nu}=\frac{\Pi_{i j}^{\mu \nu}}{\left|\vec{\Delta}_{i j}\right|^{2}}+\frac{1}{4} \sum_{l \neq i, j} \frac{n_{i l}^{\mu} n_{j l}^{\nu}+n_{i l}^{\nu} n_{j l}^{\mu}}{\left|\vec{\Delta}_{i l}\right|\left|\vec{\Delta}_{j l}\right|}, \text { for } i \neq j .
$$

Note that without loss of generality one can take $\vec{n}=\vec{e}_{p}$. Note also that $\vec{a}_{i j}=R_{i j} \cdot \vec{a}_{i j}^{\perp}$. Our next goal is to develop a systematic perturbative procedure to integrate out the offdiagonal degrees of freedom $\vec{a}_{i j}^{\perp}$. 


\subsection{The effective action to two loops and stabilization}

In this subsection we will develop a perturbative technique to integrate out the off diagonal modes $\vec{a}_{i j}^{\perp}$ and calculate the semi-classical correction to the effective action for the diagonal modes $\vec{x}_{i}$.

Numerical studies of the three matrix model [12] give a parabolic distribution of radius $R$ for the eigenvalues of one matrix, the unique three dimensional rotationally invariant lift of which is a uniform distribution inside a ball of the same radius, as argued in Appendix A.2. The radius of the distribution was found to be $R \approx 2.0>1$. This suggests a perturbative expansion in powers of $1 / R$ may prove useful. In order to proceed systematically we first rescale our variables $\vec{x}_{i}$ and $\vec{a}_{i j}$ in the following way:

$$
\vec{x}=R \tilde{\vec{x}}_{i} ; \quad \vec{a}=R \tilde{\vec{a}}_{i j} ; .
$$

Next we write the action (3.4) as:

$$
S\left[\tilde{\overrightarrow{\vec{x}}}_{i}, \tilde{\vec{a}}_{i j}^{\perp}\right]=N R^{4} \sum_{i \neq j}\left(-\frac{1}{2}\left|\tilde{\vec{\Delta}}_{i j}\right|^{2} \tilde{\vec{a}}_{i j}^{\perp} \cdot \tilde{\tilde{a}}_{j i}^{\perp}+\tilde{\Delta}_{i j}^{\mu} \tilde{a}_{j i}^{\nu}\left[\tilde{a}^{\mu}, \tilde{a}^{\nu}\right]_{j i}+\frac{1}{4}\left[\tilde{a}^{\mu}, \tilde{a}^{\nu}\right]_{i j}\left[\tilde{a}^{\mu}, \tilde{a}^{\nu}\right]_{j i}\right)
$$

Note that from a field theory point of view the parameter $1 / N R^{4}$ can be interpreted as a loop counting parameter. Next we define the correlation function:

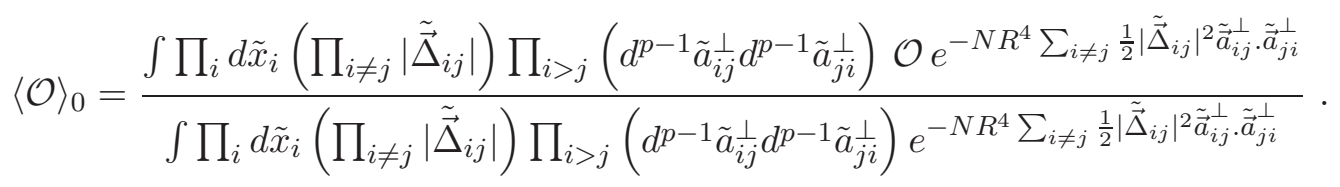

For the propagator of $\tilde{\vec{a}}_{i j}^{\perp}$ we obtain:

$$
\left\langle\tilde{a}_{i j}^{\mu \perp} \tilde{a}_{l m}^{\nu \perp}\right\rangle_{0}=\frac{1}{N R^{4}} \frac{\left(\delta^{\mu \nu}-n^{\mu} n^{\nu}\right)}{\left|\tilde{\vec{\Delta}}_{i j}\right|^{2}} \delta_{i}^{m} \delta_{j}^{l} .
$$

In deriving (3.19) one could use a frame in which $\vec{n}=\vec{e}_{p}$. Note that the cubic and quartic contributions to the action (3.17) as well as the Jacobian in the measure (3.14) depend on $\tilde{\vec{a}}_{i j}^{\perp}$ through the relation $\tilde{\vec{a}}_{i j}=R_{i j} \cdot \tilde{\vec{a}}_{i j}^{\perp}$. This is why it is convenient to calculate the two-point function:

$$
\left\langle\tilde{a}_{i j}^{\mu} \tilde{a}_{l m}^{\nu}\right\rangle_{0}=\frac{1}{N R^{4}} \frac{1}{\left|\tilde{\vec{\Delta}}_{i j}\right|^{2}} \Pi_{i j}^{\mu \nu} \delta_{i}^{m} \delta_{j}^{l}
$$

where we have used that:

$$
R_{i j}^{\mu \mu^{\prime}} \cdot\left(\delta^{\mu^{\prime} \nu^{\prime}}-n^{\mu^{\prime}} n^{\nu^{\prime}}\right) \cdot R_{i j}^{\nu \nu^{\prime}}=\delta^{\mu \nu}-n_{i j}^{\mu} n_{i j}^{\nu}=\Pi_{i j}^{\mu \nu} .
$$

We now have all the machinery required for a perturbative calculation in powers of $1 / N R^{4}$. To leading order we obtain the following first loop effective action for the diagonal modes:

$$
S_{\text {eff }}^{(1)}(R, \tilde{\vec{x}})=\left[(p-2) N^{2}-2(p-1) N+p\right] \log R+\frac{(p-2)}{2} \sum_{i \neq j} \log \left|\tilde{\vec{\Delta}}_{i j}\right|^{2} .
$$


As one can see from the first term in equation (3.22) at large $N$ and for $p \geq 2$ at one loop the effective action gives an attractive potential $V_{\text {eff }}(R)$ and is therefore not sufficient to stabilize the radius of the distribution. On the other hand the second loop corrections has an overall factor of $1 / N R^{4}$ and could balance the $\log R$ attractive potential in (3.22).

At second loop the effective action has contributions from the cubic and quartic vertices in (3.17) as well as from the quadratic term in the measure (3.14) ("ghost's contribution"). The corresponding correlation functions are: $\left\langle\left(N R^{4} \tilde{\Delta}_{i j}^{\mu} \tilde{a}_{j i}^{\nu}\left[\tilde{a}^{\mu}, \tilde{a}^{\nu}\right]_{j i}\right)^{2}\right\rangle_{0},\left\langle N R^{4} \frac{1}{4} \operatorname{tr}\left[\tilde{a}^{\mu}, \tilde{a}^{\nu}\right]^{2}\right\rangle_{0}$ and $\left\langle-2 \sum_{i, j} \tilde{\vec{a}}_{i j} \cdot D_{i j} \cdot \tilde{\vec{a}}_{j i}\right\rangle_{0}$. Using Wick contractions and the two-point function (3.20) we can calculate the second loop contribution. After somewhat tedious but straightforward calculations we obtain:

$$
\begin{aligned}
\left\langle\left(N R^{4} \sum_{i \neq j} \tilde{\Delta}_{i j}^{\mu} \tilde{a}_{j i}^{\nu}\left[\tilde{a}^{\mu}, \tilde{a}^{\nu}\right]_{j i}\right)^{2}\right\rangle_{0} & =\frac{1}{2 N R^{4}} \sum_{i, j \neq l} \frac{\left\{(4 p-6) \sin ^{2} \theta_{i, j l}+\sin ^{2} \theta_{l, i j}\right\}}{\tilde{\vec{\Delta}}_{i l}^{2} \overrightarrow{\vec{\Delta}}_{j l}^{2}} \\
\left\langle N R^{4} \frac{1}{4} \operatorname{tr}\left[\tilde{a}^{\mu}, \tilde{a}^{\nu}\right]^{2}\right\rangle_{0} & =\frac{-1}{2 N R^{4}} \sum_{i, j \neq l} \frac{\left\{(p-1)(p-2)+\sin ^{2} \theta_{l, i j}\right\}}{\tilde{\vec{\Delta}}_{i l}^{2} \tilde{\vec{\Delta}}_{j l}^{2}} \\
\left\langle-2 \sum_{i \neq j} \tilde{\vec{a}}_{i j} \cdot D_{i j} \cdot \tilde{\vec{a}}_{j i}\right\rangle_{0} & =\frac{-1}{2 N R^{4}} \sum_{i, j \neq l} \frac{2 \sin ^{2} \theta_{i, j l}}{\tilde{\vec{\Delta}}_{i l}^{2} \tilde{\vec{\Delta}}_{j l}^{2}}+O\left(\frac{1}{N}\right) .
\end{aligned}
$$

Were the angles $\theta_{l, i j}, \theta_{i, j l}$ are defined via $\cos \theta_{i, j l}=\vec{n}_{i j} \cdot \vec{n}_{i l}$ and the last term in equation (3.25) corresponds to non-planar diagram contributions, subleading in the large $N$ limit. For the total second loop contribution to the effective potential we obtain:

$$
S_{\text {eff }}^{(2)}(R, \tilde{\vec{x}})=\frac{(p-2)}{2 N R^{4}} \sum_{i, j \neq l} \frac{p-1-4 \sin ^{2} \theta_{i, j l}}{\tilde{\vec{\Delta}}_{i l}^{2} \tilde{\vec{\Delta}}_{j l}^{2}} .
$$

The full large $N$ effective action for the one loop is then

$$
S_{\text {eff }}(R, \tilde{\vec{x}})=\frac{(p-2)}{2}\left(\sum_{i \neq j} \ln \left(R^{2} \Delta_{i, j}^{2}\right)+\frac{1}{N R^{4}} \sum_{i, j \neq l} \frac{p-1-4 \sin ^{2} \theta_{i, j, l}}{\tilde{\vec{\Delta}}_{i l}^{2} \tilde{\vec{\Delta}}_{j l}^{2}}\right) .
$$

In the large $N$ limit we can trade the discrete sums in equations $(3.22),(3.26)$ for integrals over a joint distribution $\rho(\tilde{\vec{x}})$ via:

$$
\frac{1}{N} \sum_{i} \rightarrow \int_{B^{p}} d^{p} \tilde{x} \rho(\tilde{\vec{x}})
$$

where the integral is over a ball $B^{p}$ of unit radius. In the large $N$ limit we obtain the following second loop effective potential for the radius, $R$, of the distribution:

$$
V_{\mathrm{eff}}(R)=(p-2) N^{2}\left(\log R+\frac{\#(p)}{R^{4}}\right),
$$

where we have defined:

$$
\#(p)=\frac{1}{2} \int_{B^{p}} \int_{B^{p}} \int_{B^{p}} d^{p} \tilde{x} d^{p} \tilde{y} d^{p} \tilde{z} \rho(\tilde{\vec{x}}) \rho(\tilde{\vec{y}}) \rho(\tilde{\vec{z}}) \frac{p-1-4 \sin ^{2} \theta_{x, y z}}{(\tilde{\vec{x}}-\tilde{\vec{z}})^{2}(\tilde{\vec{y}}-\tilde{\vec{z}})^{2}}
$$


and $\theta_{x, y z}$ is the analog of $\theta_{i, j l}$ defined via:

$$
\cos \theta_{x, y z}=\frac{(\tilde{\vec{x}}-\tilde{\vec{y}}) \cdot(\tilde{\vec{x}}-\tilde{\vec{z}})}{|(\tilde{\vec{x}}-\tilde{\vec{y}})|(\tilde{\vec{x}}-\tilde{\vec{z}}) \mid} .
$$

We can now estimate the extent of the distribution by minimizing (3.29). We obtain:

$$
R_{p}=(4 \#(p))^{1 / 4} ; .
$$

Note that for $p \geq 5$ we have $\#(p)>0$ (the integrand in equation (3.30) is non-negative) and the ground state stabilizes at the finite radius estimated in equation (3.32). For $p=3$ numerical simulations [12] give excellent agreement with a parabolic distribution for the eigenvalues of a single matrix. As shown in Appendix A.2 the unique lift to a rotationally invariant distribution gives the uniform distribution. We therefore evaluated analytically the integral in equation (3.30), assuming a uniform distribution $\rho(\tilde{\vec{x}})=$ const (see Appendix $\mathrm{C}$ for more details). The resulting radius is:

$$
R_{3}=\left(9-\frac{3}{5} \pi^{2}\right)^{1 / 4} \approx 1.323
$$

Equations (3.29)-(3.32) contain the main result of our perturbative calculation. In the next section we discuss the gauge dependence of our expression for the radius of the distribution $R_{p}$ in equation (3.32).

\subsection{Gauge dependence}

In subsection 3.1 we outlined a change of coordinates that was equivalent to introducing the gauge $\vec{n}_{i j} \cdot \vec{a}_{i j}=0$. Alternatively we could have used standard Faddeev-Popov techniques to fix our gauge. Let us consider the gauge condition:

$$
f_{i j}=\vec{\Delta}_{i j} \cdot \vec{a}_{i j}=0
$$

The corresponding Faddeev-Popov determinant is given by:

$$
\Delta_{F P}=\prod_{i>j}\left|\vec{\Delta}_{i j}\right|^{2} \operatorname{det}\left\|\delta_{i}^{l} \delta_{j}^{m}+Y_{i j}^{\prime l m}\right\|
$$

where:

$$
Y_{i j}^{\prime l m}=\frac{\vec{n}_{i j}}{\left|\vec{\Delta}_{i j}\right|} \cdot\left(\vec{a}_{i l} \delta_{j}^{m}-\vec{a}_{m j} \delta_{i}^{l}\right)+\frac{\vec{a}_{i j} \cdot \vec{a}_{m l}}{\left|\vec{\Delta}_{i j}\right|^{2}} \cdot\left(\delta_{i}^{m}-\delta_{i}^{l}-\delta_{j}^{m}+\delta_{j}^{l}\right) .
$$

Notice that the gauge condition $f_{i j}^{\eta}=\vec{\Delta}_{i j} \cdot \vec{a}_{i j}-\eta_{i j}=0$ would result to the same FaddeevPopov determinant (3.36). Now integrating over the family of gauge functions $f_{i j}^{\eta}$ with weight $\exp \left(-\left|\eta_{i j}\right|^{2} / 2 \xi\right)$ would modify the action (3.4) to:

$$
S[X, \xi]=N \frac{1}{2} \sum_{i \neq j}\left|\vec{\Delta}_{i j}\right|^{2} a_{i j}^{\mu}\left(\Pi_{i j}^{\mu \nu}+\frac{1}{\xi} n_{i j}^{\mu} n_{i j}^{\nu}\right) a_{j i}^{\nu}-N \sum_{i \neq j} \Delta_{i j}^{\mu} a_{i j}^{\nu}\left[a^{\mu}, a^{\nu}\right]_{j i}-N \frac{1}{4} \operatorname{tr}\left[a^{\mu}, a^{\nu}\right]^{2},
$$


Next we can go through the steps considered in section 3.2, namely rescale with the radius of the joint eigenvalue distribution $R$ as in equation (3.16) and set up perturbative calculation in powers of $1 / R$. One can show that the first loop effective action $V_{\text {eff }}^{(1)}$ is still given by equation (3.22) and is thus gauge independent. However the two-point function (3.20) (the propagator for $\left.\tilde{\vec{a}}_{i j}\right)$ is modified to:

$$
\left\langle\tilde{a}_{i j}^{\mu} \tilde{a}_{l m}^{\nu}\right\rangle_{\xi}=\frac{1}{N R^{4}} \frac{1}{\left|\tilde{\vec{\Delta}}_{i j}\right|^{2}}\left(\Pi_{i j}^{\mu \nu}+\xi n_{i j}^{\mu} n_{i j}^{\nu}\right) \delta_{i}^{m} \delta_{j}^{l} .
$$

Note that the result from equation (3.20) corresponds to the choice $\xi=0$ (Landau gauge). Let us consider the choice $\xi=1$ (Feynman gauge) and calculate the second loop contribution to the effective action. Going through the same steps as in section 3.2 we obtain the analog of equation (3.29):

$$
V_{\mathrm{eff}}(R)=(p-2) N^{2}\left(\log R+\frac{\tilde{\#}(p)}{R^{4}}\right),
$$

where $\tilde{\#}(p)$ is given by:

$$
\tilde{\#}(p)=\frac{p-2}{2} \int_{B^{p}} \int_{B^{p}} \int_{B^{p}} d^{p} \tilde{x} d^{p} \tilde{y} d^{p} \tilde{z} \rho(\tilde{\overrightarrow{\vec{x}}}) \rho(\tilde{\vec{y}}) \rho(\tilde{\vec{z}}) \frac{1}{(\tilde{\vec{x}}-\tilde{\vec{z}})^{2}(\tilde{\vec{y}}-\tilde{\vec{z}})^{2}}
$$

This results in the radius:

$$
R_{p}^{\prime}=(4 \tilde{\#}(p))^{1 / 4} ; .
$$

It is clear from equation (3.40) that $\tilde{\#}(p)$ is positive for $p \geq 3$. For $p=3$ we have evaluated analytically $\tilde{\#}(3)$ (look at Appendix $\mathrm{C}$ ). The corresponding radius is:

$$
R_{3}^{\prime}=\left(\frac{9}{2}+\frac{3}{5} \pi^{2}\right)^{1 / 4} \approx 1.797
$$

Apparently the results obtained in Landau and Feynman gauges differ. In order to address the issue of gauge dependence let us focus on a particular representative of the family of gauge conditions $f_{i j}^{\eta}=\vec{\Delta}_{i j} \cdot \vec{a}_{i j}-\eta_{i j}=0$. Note that the change of coordinates (3.11) considered in section 3.2 implements the $\eta_{i j}=0$ case. One can show that the gauge condition for general $\eta_{i j}$ can be implemented along the lines of section 3.2 via the following modified change of coordinates:

$$
X^{\mu}=U \| x_{i}^{\mu} \delta_{i j}+R_{i j}^{\mu \nu} a_{i j}^{\nu \perp}+\frac{n_{i j}^{\mu}}{\left|\vec{\Delta}_{i j}\right|} \eta_{i j}|| U^{-1} ; \quad U \in S U(N) ; .
$$

Let us suppose that the theory has settled in its ground state which is a commuting phase. There should exist unitary matrix $V \in S U(N)$ which simultaneously diagonalizes the $X^{\mu}$ matrices and hence we can write:

$$
V^{-1} \lambda^{\mu} V=U\left\|x_{i}^{\mu} \delta_{i j}+R_{i j}^{\mu \nu} a_{i j}^{\nu \perp}+\frac{n_{i j}^{\mu}}{\left|\vec{\Delta}_{i j}\right|} \eta_{i j}\right\| U^{-1},
$$


where $\lambda^{\mu}$ is a diagonal matrix. Now if we square equation (3.44), take a trace over the gauge indices and sum over $\mu$ we obtain:

$$
\sum_{i} \vec{\lambda}_{i}^{2}=\sum_{i} \vec{x}_{i}^{2}+\sum_{i \neq j}\left|\vec{a}_{i j}^{\perp}\right|^{2}+\sum_{i \neq j} \frac{\left|\eta_{i j}\right|^{2}}{\left|\vec{\Delta}_{i j}\right|^{2}} \geq \sum_{i} \vec{x}_{i}^{2}+\sum_{i \neq j} \frac{\left|\eta_{i j}\right|^{2}}{\left|\vec{\Delta}_{i j}\right|^{2}} .
$$

Next we define average radii of the distribution $r_{\lambda}$ and $r_{x}$ via:

$$
r_{\lambda}^{2}=\frac{1}{N} \sum_{i} \vec{\lambda}_{i}^{2} ; \quad r_{x}^{2}=\frac{1}{N} \sum_{i} \vec{x}_{i}^{2}
$$

and learn that:

$$
r_{\lambda}^{2}-r_{x}^{2} \geq \frac{1}{N} \sum_{i \neq j} \frac{\left|\eta_{i j}\right|^{2}}{\left|\vec{\Delta}_{i j}\right|^{2}}
$$

Therefore the average radius of the eigenvalue distribution $r_{\lambda}$ always differs from the average radius of the distribution of the diagonal modes $r_{x}$, unless $\eta_{i j}=0$ or the eigenvalues are infinitely spread in which case there is no well defined average radius. This could explain why the gauge fixing procedure outlined above, which involved averaging over all possible values of $\eta_{i j}$ failed to produce a gauge independent answer for the radius of the eigenvalue distribution. These consideration suggests that the gauge $\eta_{i j}=0$ should be optimal for describing the almost commuting theory.

Alternatively one could take the point of view that both gauge choices are equally valid and describe different approximations to the true result, they only differ due to the intrinsic errors in a perturbative calculation. If we take this point of view we can use the difference to estimate the errors in our estimate of $R$. If we do this we conclude that $R \sim 1.6 \pm 0.5$ which is in reasonable agreement with the numerical results.

\section{Discussion}

In this paper we have followed two threads, in the first we investigated the 3-matrix model of $[5,7]$ and find that in the large $g$ limit the 3 -matrices commute and have a joint eigenvalue

distribution given by the uniform distribution within a ball of radius $R=\left(\frac{3 \pi}{2 g}\right)^{1 / 3}$. We show that a simple effective potential for the radius of the distribution reproduces the exact result. Furthermore, in Appendix A we first demonstrated how to do perturbation theory around the uniform distribution and then how this distribution is the unique rotational invariant lift of the eigenvalue distribution of a single matrix.

Encouraged by the success of this effective potential calculation we develop an effective potential for the radius of the $p$-component Yang-Mills matrix model to two loops. We have done this by deriving an effective potential for the diagonal modes while preserving $S O(3)$ invariance and then assuming that these modes are uniformly distributed. The direct analog of the computations in earlier sections would be a two loop computation in the axial gauge (where one of the matrices is diagonalized). Unfortunately this gauge choice leads to infrared divergences at two loops and so we have not pursued this option.

We found that it is necessary to go to two loops as at one loop the effective potential is not stable since there is no classical potential and the one loop term gives an attractive 
potential which is a rotationally invariant version of the Vandermonde determinant. The eigenvalue repulsion arises at two loops and gives a $\frac{1}{R^{4}}$ hard core potential. It is easy to see that higher order terms give inverse higher powers of $R$ and our two loop estimate can only be a very rough approximation.

Our estimate for the radius is unfortunately gauge dependent with $R=1.323$ in the Landau gauge and $R=1.797$ in the Feynman gauge. It is reasonable to assume that the difference between these is an indication of the errors in the method which would indicate that perhaps a reasonable estimate can be obtained by averaging the two and taking the difference as an indication of the error yielding the prediction $R=1.6 \pm 0.5$. An alternative approach pursued by Hotta, Nishimura and Tsuchiya [15] examined similar questions, for some observables, in the general Yang-Mills $p$-matrix model ${ }^{2}$.

If we take their result

$$
<\frac{\operatorname{tr}}{N}\left(X_{a}^{2}\right)>=\sqrt{\frac{p}{2}}\left(1+\frac{7}{6 p}+\cdots\right)
$$

and assume that this is valid for $p=3$ together with the assumption that the eigenvalue distribution of a single matrix is parabolic of extent $R$ (which is consistent with a uniform joint distribution for commuting matrices) then $\left\langle\frac{\operatorname{tr}}{N}\left(X_{a}^{2}\right)>=3 \frac{R^{2}}{5}\right.$ and using (4.1) we obtain the estimate $R=1.68$, which is surprisingly close to the estimate we obtain above. In this case one can also attempt an estimate of the error by noting that if instead of (4.1) we use $<\frac{\operatorname{tr}}{N}\left(X_{a}^{2}\right)>=\sqrt{\frac{p}{2}}\left(\frac{1}{\left(1-\frac{7}{6 p}\right)}+\cdots\right)$ which has the same leading large $p$ expansion we obtain $R=1.83$ and therefore estimate the error (within the assumption of a parabolic distribution) that $R=1.75 \pm .15$ which is in surprisingly good agreement with Monte Carlo simulations [12] which give a value for $R=2.0$

We conclude that, though the random matrices are not commuting, a useful approximation is to take the background formed by these fluctuating matrices as that of commuting matrices whose joint eigenvalue distribution is approximately uniform within a ball of radius $R$. This background gives reasonable agreement with the numerical work and serves as a reasonable starting point for further work.

\section{Acknowledgments}

The work of V. F. was funded by an INSPIRE IRCSET-Marie Curie International Mobility Fellowship.

\section{A Corrections at large coupling and lifting the distribution}

In this appendix we provide a more rigorous derivation of the uniform distribution obtained in section 2.2. We first develop perturbation theory around the uniform background and then obtain the leading correction to the uniform distribution for large coupling.

\footnotetext{
${ }^{2}$ Hotta et al [15] also obtained a two loop effective action for eigenvalues of the matrices $X_{\mu}$ by integrating out the $U(N)$ transformations that diagonalize the matrices. This yields a non-rotationally invariant effective action, which does not lead to a stable effective potential for the radial extent of the eigenvalues.
} 
In the second section we show that the uniform distribution is the unique rotationally invariant lift of the parabolic distribution and more generally establish the rotational invariant lifts of a one dimensional distribution to three and then down to two dimensions.

\section{A.1 Iterative Solution}

Here we derive an integral equation of the second kind for the correction to the uniform distribution (2.31). Our starting point is the integral equation (2.37):

$$
1=\int d^{3} x^{\prime} \frac{\rho\left(\vec{x}^{\prime}\right)}{\left|\vec{x}-\vec{x}^{\prime}\right|^{2}\left(1+g^{2}\left|\vec{x}-\vec{x}^{\prime}\right|^{2}\right)}, \quad \text { for }|\vec{x}| \leq R,
$$

where the integral is performed over a ball of radius $R$. Since the radius is $g$ dependent (with leading order dependence given by equation (2.33) ) it is more convenient to introduce new variables:

$$
\vec{\eta}=\vec{x} / R, \quad \tilde{\rho}(\vec{\eta})=R \rho(R \vec{\eta}) .
$$

In these variables the integral equation (A.1) becomes:

$$
1=\int d^{3} \eta^{\prime} \frac{\tilde{\rho}\left(\vec{\eta}^{\prime}\right)}{\left|\vec{\eta}-\vec{\eta}^{\prime}\right|^{2}\left(1+(R g)^{2}\left|\vec{\eta}-\vec{\eta}^{\prime}\right|^{2}\right)}, \quad \text { for }|\vec{\eta}| \leq 1
$$

where the integral is performed over a ball of unit radius. Note that from equation (2.33) it follows that at large $g, R g \sim g^{2 / 3}$ and $R g$ is also large. Therefore we are interested in solving equation (A.3) at large $R g$. The fact that the kernel of equation (A.3) is a delta convergent series:

$$
\frac{1}{\left|\vec{\eta}-\vec{\eta}^{\prime}\right|^{2}\left(1+(R g)^{2}\left|\vec{\eta}-\vec{\eta}^{\prime}\right|^{2}\right)}=\frac{2 \pi^{2}}{R g} \delta\left(\vec{\eta}-\vec{\eta}^{\prime}\right)+\ldots
$$

suggests that we make the following definitions:

$$
\Delta K\left(\vec{\eta}-\vec{\eta}^{\prime}, R g\right)=\frac{R g}{2 \pi^{2}\left|\vec{\eta}-\vec{\eta}^{\prime}\right|^{2}\left(1+(R g)^{2}\left|\vec{\eta}-\vec{\eta}^{\prime}\right|^{2}\right)}-\delta\left(\vec{\eta}-\vec{\eta}^{\prime}\right), \quad \Delta \tilde{\rho}(\vec{\eta})=\tilde{\rho}(\vec{\eta})-\frac{R g}{2 \pi^{2}} .
$$

Note that $R g /\left(2 \pi^{2}\right)$ corresponds to the uniform distribution (2.31) in the variables (A.2), which means that $\Delta \rho$ is the correction to the uniform distribution in the same variables. It is straightforward to write the integral equation (A.3) in terms of $\Delta K$ and $\Delta \tilde{\rho}$ :

$$
\Delta \tilde{\rho}(\eta)=-\frac{R g}{2 \pi^{2}} \int d^{3} \eta^{\prime} \Delta K\left(\vec{\eta}-\vec{\eta}^{\prime}, R g\right)-\int d^{3} \eta^{\prime} \Delta K\left(\vec{\eta}-\vec{\eta}^{\prime}, R g\right) \Delta \tilde{\rho}\left(\vec{\eta}^{\prime}\right) .
$$

Equation (A.6) is an integral equation of the second kind for the function $\Delta \tilde{\rho}$ and can be developed recursively as the series:

$$
\Delta \tilde{\rho}=-\frac{R g}{2 \pi^{2}}\left[\int \Delta K-\int \Delta K \int \Delta K+\int \Delta K \int \Delta K \int \Delta K-\ldots\right],
$$

where we used condensed notations. One can check that $\int d^{3} \eta^{\prime} \Delta K \sim 1 /(R g)$ for $|\vec{\eta}|<1$, which suggests that the series is convergent for large $R g$. This can be verified numerically. 
If the series is convergent this is sufficient for the integral equation (A.6) to have a unique solution given by (A.7).

Here we present the first iteration (the leading order) correction to the uniform distribution defined by:

$$
\tilde{\rho}_{(1)}(\eta, R g)=-\frac{R g}{2 \pi^{2}} \int d^{3} \eta^{\prime} \Delta K\left(\vec{\eta}-\vec{\eta}^{\prime}\right)
$$

Our result is:

$$
\begin{aligned}
\tilde{\rho}_{(1)}(R g, \eta) & =\frac{R g}{2 \pi^{3}}\left[\pi-\tan ^{-1}[R g(1+\eta)]-\tan ^{-1}[R g(1-\eta)]\right]-\frac{1}{8 \pi^{3} \eta} \log \left[\frac{1+(R g)^{2}(1-\eta)^{2}}{1+(R g)^{2}(1+\eta)^{2}}\right] \\
& -\frac{(R g)^{2}}{8 \pi^{3} \eta}\left(1-\eta^{2}\right) \log \left[\frac{(1+\eta)^{2}\left(1+(R g)^{2}(1-\eta)^{2}\right)}{(1-\eta)^{2}\left(1+(R g)^{2}(1+\eta)^{2}\right)}\right] .
\end{aligned}
$$

Note that $\tilde{\rho}_{1}(\eta, R g)$ is regular and of order one for $\eta \in[0,1)$. One can check that $\int d^{3} \eta \tilde{\rho}_{(1)}(\eta, R g) \sim \log (R g)$, which is subleading relative to the uniform distribution $R g /\left(2 \pi^{2}\right)$ whose integral is of order $R g$.

We can perform a non-trivial check of this results by calculating the observable:

$$
\nu=\frac{1}{3} g^{2} \int d^{3} x \rho(\vec{x}) \vec{x}^{2}=\frac{1}{3} R^{2}(R g)^{2} \int d^{3} \eta \tilde{\rho}(\vec{\eta}) \vec{\eta}^{2},
$$

obtained in closed form in ref. [7]. Note that the radius $R$ can be extracted from $\tilde{\rho}(\eta)$ through the relation:

$$
\int d^{3} \eta \tilde{\rho}(\eta)=R^{-2}
$$

Using the leading order result for $\tilde{\rho}$ :

$$
\tilde{\rho}(\eta, R g)=\frac{R g}{2 \pi^{2}}+\tilde{\rho}_{(1)}(\eta, R g)+O\left((R g)^{-1}\right),
$$

it is straightforward to obtain:

$$
\begin{aligned}
& R^{-2}=\frac{2 R g}{3 \pi}+\frac{2 \log (2 R g)+1}{2 \pi^{2}}+\ldots \\
& \int d^{3} \eta \tilde{\rho}(\vec{\eta}) \vec{\eta}^{2}=\frac{2 R g}{5 \pi}+\frac{2 \log (2 R g)-1}{2 \pi^{2}}+\ldots
\end{aligned}
$$

substituting equations (A.13) and (A.14) in equation (A.10), we can obtain large $R g$ expansion of $\nu$. Furthermore from equation (A.14) we can obtain the large $g$ expansion of $R g$. Therefore, we can calculate the first two leading terms in the large $g$ expansion of $\nu$ :

$$
\nu=\frac{(12 \pi)^{2 / 3}}{20} g^{4 / 3}-\frac{(12 \pi)^{1 / 3}}{4 \pi} g^{2 / 3}+\ldots
$$

which is exactly the result of ref. [7]. 


\section{A.2 "Lifting" the 1d distribution}

In this subsection we derive a relation between the two and three dimensional distributions considered in section 2.2 and the one dimensional distribution considered in section $2.1 \mathrm{We}$ begin by discussing the lift to the three dimensional distribution.

Let us consider equation (2.26), if we apply the operator $\vec{n} . \nabla_{x}$ on both sides of the equation we obtain:

$$
\vec{n} \cdot \vec{x}=\int \frac{d^{3} x^{\prime} \rho\left(\vec{x}^{\prime}\right)}{\left(\vec{n} \cdot\left(\vec{x}-\vec{x}^{\prime}\right)\right)\left(1+g^{2}\left(\vec{n} \cdot\left(\vec{x}-\vec{x}^{\prime}\right)\right)^{2}\right)}
$$

Next if we define $u=\vec{n} . \vec{x}$, choose a coordinate frame in the integral along $\vec{x}^{\prime}$ in which we have $\vec{n} \cdot \vec{x}^{\prime}=x^{\prime 1}$ and define:

$$
\rho_{1}\left(x_{1}\right)=\int_{-\sqrt{R^{2}-x_{1}^{2}}}^{\sqrt{R^{2}-x_{1}^{2}}} d x_{2} \int_{-\sqrt{R^{2}-x_{1}^{2}-x_{2}^{2}}}^{\sqrt{R^{2}-x_{1}^{2}-x_{2}^{2}}} d x_{3} \rho(\vec{x}),
$$

we obtain equation (2.14):

$$
u=\int \frac{d x^{1} \rho_{1}\left(x^{\prime 1}\right)}{\left(u-x^{\prime 1}\right)\left(1+g^{2}\left(u-x^{1}\right)^{2}\right)},
$$

which we solved in section 2.1 at large $g$ to obtain the parabolic distribution (2.18) of ref. [13]. To find the corresponding three dimensional ("lifted") distribution we have to solve the integral equation (A.17). This can easily be achieved by integrating over the disc $x_{2}^{2}+x_{3}^{2} \leq R^{2}-x_{1}^{2}$ in cylindrical coordinates:

$$
\rho_{1}\left(x_{1}\right)=\int_{0}^{\sqrt{R^{2}-x_{1}^{2}}} d r(2 \pi r) \rho\left(\sqrt{x_{1}^{2}+r^{2}}\right)=\int_{x_{1}}^{R} d w(2 \pi w) \rho(w),
$$

where we substituted $r=\sqrt{w^{2}-x_{1}^{2}}$. By differentiating ${ }^{3}$ equation (A.19) with respect to $x_{1}$ we can solve for $\rho$ in therms of $\rho_{1}$ :

$$
\rho(x)=-\frac{\rho_{1}^{\prime}(x)}{2 \pi x} .
$$

Equation (A.20) is the unique solution for a "lifted" rotationally invariant three dimensional distribution reproducing the one dimensional distribution $\rho_{1}$. Furthermore since at large $g$ the three matrix model is commuting this suggests that the lifted distribution is the joint eigenvalue distribution of the model. It is easy to check that if one substitutes the parabolic distribution (2.18) with the radius (2.19) into equation (A.20) one obtains exactly the uniform distribution (2.31):

$$
\rho(\vec{x})=\frac{g}{2 \pi^{2}} .
$$

\footnotetext{
${ }^{3}$ Note that there are no subtleties in differentiating near the boundary since $\rho_{1}(R)=0$.
} 
We can now reduce to the two dimensional distribution by integrating over the additional coordinate.

$$
\rho_{2}(\vec{r})=-\int_{-\sqrt{R^{2}-r^{2}}}^{\sqrt{R^{2}-r^{2}}} \frac{\rho_{1}^{\prime}\left(\sqrt{\left.r^{2}+z^{2}\right)}\right.}{2 \pi \sqrt{r^{2}+z^{2}}} d z,
$$

which in the large $g$ limit reproduces (2.20).

\section{B The Jacobian}

In this section we outline the calculation of the Jacobian (3.12). Let us begin by differentiating equation (3.11). We obtain:

$$
\left(U^{-1} d \vec{X} U\right)_{i j}=d \vec{x}_{i} \delta_{i j}+d R_{i j} \cdot \vec{a}_{i j}^{\perp}+R_{i j} \cdot d \vec{a}_{i j}^{\perp}-\left|\vec{\Delta}_{i j}\right| \vec{n}_{i j} \theta_{i j}-[a, \theta]_{i j},
$$

where we have defined the Maurer-Cartan form $\theta \equiv U^{-1} d U$. Next we define the tetrads $\vec{E}$ via:

$$
\vec{E}_{i}=\left(U^{-1} d \vec{X} U\right)_{i i} ; \quad \vec{E}_{i j}=\left(U^{-1} d \vec{X} U\right)_{i j} \quad \text { for } \quad i \neq j ; .
$$

In matrix form we have:

\begin{tabular}{|c|c|c|c|}
\hline & $d \vec{x}_{k}$ & $R_{r s} \cdot d \vec{a}_{r s}^{\perp}$ & $d u_{l m}$ \\
\hline$\vec{E}_{i}$ & $\hat{1} \delta_{i}^{k}$ & 0 & $-\frac{\delta[\vec{a}, \theta]_{i i}}{\delta u_{l m}}$ \\
\hline$\vec{E}_{i j}^{\perp}$ & $\prod_{i j} \cdot \frac{\partial R_{i j}}{\partial \vec{x}_{k}} \cdot \vec{a}_{i j}^{\perp}$ & $\hat{1} \delta_{i}^{r} \delta_{j}^{s}$ & $-\Pi_{i j} \cdot \frac{\delta[\vec{a}, \theta]_{i j}}{\delta u_{l m}}$ \\
\hline$E_{i j}^{\|}$ & $\frac{\partial \vec{n}_{i j}}{\partial \vec{x}_{k}} \cdot \vec{a}_{i j}$ & 0 & $\left|\vec{\Delta}_{i j}\right| \frac{\delta \theta_{i j}}{\delta u_{l m}}+\vec{n}_{i j} \cdot \frac{\delta[\vec{a}, \theta]_{i j}}{\delta u_{l m}}$ \\
\hline
\end{tabular}

where $\Pi_{i j}=\hat{1}-\vec{n}_{i j} \vec{n}_{i j}$ and we have split the off-diagonal tetrads $\vec{E}_{i j}$ into:

$$
\vec{E}_{i j}^{\perp}=\Pi_{i j} \cdot \vec{E}_{i j} ; \quad \text { and } \quad E_{i j}^{\|}=-\vec{n}_{i j} \cdot \vec{E}_{i j} ; .
$$

We have also used that:

$$
\vec{n}_{i j} \cdot \frac{\partial R_{i j}}{\partial x_{k}}+\frac{\partial \vec{n}_{i j}}{\partial x_{k}} \cdot R_{i j}=0 .
$$

Now the Jacobian of interest, $J$, is given by the determinant of $\|E\|$. It is an easy exercise to show that the Jacobian is given by:

$$
J=\operatorname{det}||\left|\vec{\Delta}_{i j}\right| \frac{\delta \theta_{i j}}{\delta u_{l m}}+\vec{n}_{i j} \cdot \frac{\delta[\vec{a}, \theta]_{i j}}{\delta u_{l m}}+\sum_{k} \frac{\partial \vec{n}_{i j}}{\partial x_{k}} \cdot \vec{a}_{i j} \frac{\delta[\vec{a}, \theta]_{k k}}{\delta u_{l m}} \|
$$


One can show that the determinant in equation (B.5) factorizes:

$$
J=\operatorname{det}\left\|\left|\vec{\Delta}_{i j}\right| \delta_{i}^{r} \delta_{j}^{s}+\vec{n}_{i j} \cdot \frac{\delta[\vec{a}, \theta]_{i j}}{\delta \theta_{r s}}+\sum_{\mu, k} \frac{\partial \vec{n}_{i j}}{\partial x_{k}^{\mu}} \cdot \vec{a}_{i j} \frac{\delta\left[a^{\mu}, \theta\right]_{k k}}{\delta \theta_{r s}}\right\| \operatorname{det}\left\|\frac{\delta \theta_{r s}}{\delta u_{l m}}\right\|
$$

The last determinant in equation (B.6) produces the correct measure of $S U(N)$ and it is the first part $J^{\prime}$ that we are really interested in. One can verify that:

$$
J^{\prime}=\left(\prod_{i \neq j}\left|\vec{\Delta}_{i j}\right|\right) \operatorname{det}|| \delta_{i}^{l} \delta_{j}^{m}+Y_{i j}^{l m} \|
$$

where $Y_{i j}^{l m}$ is given by:

$$
Y_{i j}^{l m}=\frac{\vec{n}_{i j}}{\left|\vec{\Delta}_{i j}\right|} \cdot\left(\vec{a}_{i l} \delta_{j}^{m}-\vec{a}_{m j} \delta_{i}^{l}\right)+\frac{\vec{a}_{i j} \cdot \Pi_{i j} \cdot \vec{a}_{m l}}{\left|\vec{\Delta}_{i j}\right|^{2}} \cdot\left(\delta_{i}^{m}-\delta_{i}^{l}-\delta_{j}^{m}+\delta_{j}^{l}\right),
$$

where we have used that:

$$
\frac{\delta[\vec{a}, \theta]_{i j}}{\delta \theta_{l m}}=\left(\vec{a}_{i l} \delta_{j}^{m}-\vec{a}_{m j} \delta_{i}^{l}\right) ; \quad \frac{\partial n_{i j}^{\mu}}{\partial x_{k}^{\nu}}=\frac{\Pi_{i j}^{\mu \nu}}{\left|\vec{\Delta}_{i j}\right|}\left(\delta_{i}^{k}-\delta_{j}^{k}\right) ; \quad \vec{a}_{i j} . \Pi_{i j}=\vec{a}_{i j} ; .
$$

Now using that:

$$
\operatorname{det}\left\|\delta_{i}^{l} \delta_{j}^{m}+Y_{i j}^{l m}\right\|=\exp \left\{\operatorname{tr} Y-\frac{1}{2} \operatorname{tr} Y^{2}+O\left(Y^{3}\right)\right\}
$$

One can easily verify that:

$$
J^{\prime}=\exp \left\{\sum_{i \neq j} \log \left|\vec{\Delta}_{i j}\right|-2 \sum_{i \neq j} \overline{\vec{a}}_{i j} \cdot \hat{D}_{i j} \cdot \vec{a}_{j i}+O\left(a^{3}\right)\right\},
$$

where $\hat{D}_{i j}$ is given by:

$$
D_{i j}^{\mu \nu}=\frac{\Pi_{i j}^{\mu \nu}}{\left|\vec{\Delta}_{i j}\right|^{2}}+\frac{1}{4} \sum_{k} \frac{n_{i k}^{\mu} n_{j k}^{\nu}+n_{i k}^{\nu} n_{j k}^{\mu}}{\left|\vec{\Delta}_{i k}\right|\left|\vec{\Delta}_{j k}\right|} .
$$

Therefore our final expression for the measure is:

$$
\mu=D U \prod_{i} d x_{i} \prod_{i>j}\left(d^{p-1} a_{i j}^{\perp} d^{p-1} a_{j i}^{\perp}\right) \exp \left\{\sum_{i \neq j} \log \left|\vec{\Delta}_{i j}\right|-2 \sum_{i \neq j} \overline{\vec{a}}_{i j} \cdot \hat{D}_{i j} \cdot \vec{a}_{j i}+O\left(a^{3}\right)\right\},
$$

\section{The constants \#(p) and $\tilde{\#}(p)$}

In this section we will provide details about the analytic evaluation of the quantities \# $(p)$ and $\tilde{\#}(p)$ defined in equations (3.30) and (3.40) respectively. Note that one can write:

$$
\#(p)=\frac{p-5}{2} A_{p}+2 C_{p} ; \quad \tilde{\#}(p)=\frac{p-2}{2} A_{p} ;,
$$


where:

$$
\begin{aligned}
A_{p} & =\int_{B^{p}} \int_{B^{p}} \int_{B^{p}} d^{p} \tilde{x} d^{p} \tilde{y} d^{p} \tilde{z} \rho(\tilde{\vec{x}}) \rho(\tilde{\vec{y}}) \rho(\tilde{\vec{z}}) \frac{1}{(\tilde{\vec{x}}-\tilde{\vec{z}})^{2}(\tilde{\vec{y}}-\tilde{\tilde{z}})^{2}} \\
C_{p} & =\int_{B^{p}} \int_{B^{p}} \int_{B^{p}} d^{p} \tilde{x} d^{p} \tilde{y} d^{p} \tilde{z} \rho(\tilde{\vec{x}}) \rho(\tilde{\vec{y}}) \rho(\tilde{\vec{z}}) \frac{\cos ^{2} \theta_{x, y z}}{(\tilde{\vec{x}}-\tilde{\vec{z}})^{2}(\tilde{\vec{y}}-\tilde{\vec{z}})^{2}} .
\end{aligned}
$$

Let us consider first the quantity $A_{p}$. Note that the integrals along $\tilde{x}$ and $\tilde{y}$ in equation (C.2) factorize and one can write:

$$
A_{p}=\int_{B^{p}} d^{p} \tilde{z} \rho(\tilde{\vec{z}}) Q_{p}(|\tilde{\vec{z}}|)^{2}=\frac{2 \pi^{p / 2}}{\Gamma(p / 2)} \int_{0}^{1} d \tilde{z} \tilde{z}^{p-1} \rho(\tilde{z}) Q_{p}(\tilde{z})^{2}
$$

where:

$$
Q_{p}(\tilde{z}) \equiv \int_{B^{p}} d^{p} \tilde{x} \rho(\tilde{x}) \frac{1}{(\tilde{\vec{x}}-\tilde{\vec{z}})^{2}}
$$

and we have used that the distribution is $S O(p)$ symmetric (namely $\rho(\tilde{\vec{x}})=\rho(\tilde{x})$ ). Note that for a uniform distribution we have $\rho(\tilde{x})=p \Gamma(p / 2) / 2 \pi^{p / 2}$ and we have:

$$
A_{p}=p \int_{0}^{1} d \tilde{z} \tilde{z}^{p-1} Q_{p}(\tilde{z})^{2}
$$

One can show that for $p=3$ and a uniform distribution $Q_{3}(\tilde{z})$ is given by:

$$
Q_{3}(\tilde{z})=\frac{3}{2}\left[1+\frac{1-\tilde{z}^{2}}{z} \operatorname{Arctanh}(\tilde{z})\right]
$$

and

$$
A_{3}=3 \int_{0}^{1} d \tilde{z} \tilde{z}^{2} Q_{3}(\tilde{z})^{2}=\frac{3}{20}\left(15+2 \pi^{2}\right) .
$$

Let us now focus on the quantity $C_{p}$. After using equation (3.31) and the $S O(p)$ symmetry of the eigenvalue distribution, one can show that:

$$
\begin{aligned}
C_{p} & =\frac{3}{4} A_{p}-\int_{B^{p}} \int_{B^{p}} \int_{B^{p}} d^{p} \tilde{x} d^{p} \tilde{y} d^{p} \tilde{z} \rho(\tilde{\vec{x}}) \rho(\tilde{\vec{y}}) \rho(\tilde{\vec{z}}) \frac{(\tilde{\vec{x}}-\tilde{\vec{z}}) \cdot(\tilde{\vec{y}}-\tilde{\tilde{z}})}{(\tilde{\vec{x}}-\tilde{\vec{z}})^{2}(\tilde{\vec{y}}-\tilde{\tilde{z}})^{4}} \\
& =\frac{3}{4} A_{p}+\frac{2 \pi^{p / 2}}{\Gamma(p / 2)} \int_{0}^{1} d \tilde{z} \tilde{z}^{p-1} \rho(\tilde{z}) Q_{p}^{\prime}(\tilde{z}) \Phi_{p}^{\prime}(\tilde{z})
\end{aligned}
$$

where

$$
\Phi_{p}(\tilde{z}) \equiv \frac{1}{2} \int_{B^{p}} d^{p} \tilde{x} \rho(\tilde{x}) \log |(\tilde{\vec{x}}-\tilde{\vec{z}})| .
$$

For an uniform distribution $\rho(\tilde{z})$ we have:

$$
C_{p}=\frac{3}{4} A_{p}+p \int_{0}^{1} d \tilde{z} \tilde{z}^{p-1} Q_{p}^{\prime}(\tilde{z}) \Phi_{p}^{\prime}(\tilde{z})
$$


One can show that for $p=3$ and a uniform distribution $\Phi_{3}(\tilde{z})$ is given by:

$$
\Phi_{3}(\tilde{z})=\frac{1}{96 \tilde{z}}\left[-34 \tilde{z}+6 \tilde{z}^{3}+3(\tilde{z}-1)^{3}(3+\tilde{z}) \log |1-\tilde{z}|-3(\tilde{z}-3)(1+\tilde{z})^{3} \log |1+\tilde{z}|\right]
$$

and one can calculate $C_{3}$ :

$$
C_{3}=\frac{3}{20}\left(15+\frac{1}{2} \pi^{2}\right)
$$

Now one can substitute the results from equations (C.8) and (C.14) into equation (C.1) to obtain:

$$
\#(3)=\frac{3}{20}\left(15-\pi^{2}\right) ; \quad \tilde{\#}(3)=\frac{3}{40}\left(15+2 \pi^{2}\right) ;,
$$

\section{References}

[1] R. Dijkgraaf, E. P. Verlinde and H. L. Verlinde, "Matrix string theory," Nucl. Phys. B 500 (1997) 43 [hep-th/9703030].

[2] N. Ishibashi, H. Kawai, Y. Kitazawa and A. Tsuchiya, "A Large N reduced model as superstring," Nucl. Phys. B 498 (1997) 467 [hep-th/9612115].

[3] A. Connes, M. R. Douglas and A. S. Schwarz, "Noncommutative geometry and matrix theory: Compactification on tori," JHEP 9802 (1998) 003 [hep-th/9711162].

[4] T. Banks, W. Fischler, S. H. Shenker and L. Susskind, "M theory as a matrix model: A Conjecture," Phys. Rev. D 55 (1997) 5112 [hep-th/9610043].

[5] Hoppe, J. R. 1982, Ph.D. Thesis.

[6] D. E. Berenstein, J. M. Maldacena and H. S. Nastase, "Strings in flat space and pp waves from N=4 Super Yang Mills," AIP Conf. Proc. 646 (2003) 3.

[7] V. A. Kazakov, I. K. Kostov and N. A. Nekrasov, "D-particles, matrix integrals and KP hierarchy," Nucl. Phys. B 557, 413 (1999) [arXiv:hep-th/9810035].

[8] R. Delgadillo-Blando, D. O'Connor and B. Ydri, "Geometry in Transition: A Model of Emergent Geometry," Phys. Rev. Lett. 100 (2008) 201601 [arXiv:0712.3011 [hep-th]].

[9] H. Steinacker, "Gravity and compactified branes in matrix models," JHEP 1207 (2012) 156 [arXiv:1202.6306 [hep-th]].

[10] D. N. Blaschke and H. Steinacker, "Schwarzschild Geometry Emerging from Matrix Models," Class. Quant. Grav. 27 (2010) 185020 [arXiv:1005.0499 [hep-th]].

[11] C. T. Asplund, D. Berenstein and E. Dzienkowski, "Large N classical dynamics of holographic matrix models," arXiv:1211.3425 [hep-th].

[12] R. Delgadillo-Blando and D. O'Connor, "Matrix geometries and Matrix Models," JHEP 1211 (2012) 057 [arXiv:1203.6901 [hep-th]].

[13] D. E. Berenstein, M. Hanada and S. A. Hartnoll, "Multi-matrix models and emergent geometry," JHEP 0902, 010 (2009) [arXiv:0805.4658 [hep-th]].

[14] R. C. Myers, "Dielectric branes," JHEP 9912 (1999) 022 [hep-th/9910053].

[15] T. Hotta, J. Nishimura and A. Tsuchiya, "Dynamical aspects of large N reduced models," Nucl. Phys. B 545 (1999) 543 [hep-th/9811220]. 
[16] T. Azuma, K. Nagao and J. Nishimura, "Perturbative dynamics of fuzzy spheres at large N," JHEP 0506 (2005) 081 [hep-th/0410263].

[17] R. Delgadillo-Blando, D. O'Connor and B. Ydri, "Matrix Models, Gauge Theory and Emergent Geometry," JHEP 0905, 049 (2009) [arXiv:0806.0558 [hep-th]].

[18] D. O'Connor and B. Ydri, "Monte Carlo Simulation of a NC Gauge Theory on The Fuzzy Sphere," JHEP 0611 (2006) 016 [hep-lat/0606013].

[19] T. Azuma, S. Bal, K. Nagao and J. Nishimura, "Nonperturbative studies of fuzzy spheres in a matrix model with the Chern-Simons term," JHEP 0405 (2004) 005 [hep-th/0401038].

[20] T. Azuma, S. Bal and J. Nishimura, "Dynamical generation of gauge groups in the massive Yang-Mills-Chern-Simons matrix model," Phys. Rev. D 72 (2005) 066005 [hep-th/0504217]. 